Pacific Journal of Mathematics

FUNCTIONAL RELATIONSHIPS BETWEEN A SUBNORMAL
OPERATOR AND ITS MINIMAL NORMAL EXTENSION 


\title{
FUNCTIONAL RELATIONSHIPS BETWEEN A SUBNORMAL OPERATOR AND ITS MINIMAL NORMAL EXTENSION
}

\author{
ROBERT F. OLIN
}

Let $K$ be a compact subset of the plane. $C(K)$ denotes the continuous functions on $K$ and $R(K)$ denotes those continuous functions of $K$ which are uniform limits of rational functions whose poles lie off $K$. We say that $f$ is minimal on $K$ if $f \in R(K)$ and for every complex number $c$

$$
R\left(L_{c}\right)=C\left(L_{c}\right)
$$

where $L_{c}=\{z \in K \mid(f z)=c\}$.

Let $S$ be a subnormal operator on a Hilbert space $\mathscr{C}$ with its minimal normal extension $N$ on the Hilbert space $\mathscr{K}$. The spectrum of $S$ is denoted by $\sigma(S)$. In this paper it is shown that if $f$ is minimal on $\sigma(S)$ then $f(N)$ on $\mathscr{K}$ is the minimal normal extension of $f(N)$ restricted to $\mathscr{C}$. Some new results about subnormal operators follow as corollaries of this theorem.

An operator $S$ acting on a Hilbert space $\mathscr{H}$ is called subnormal if there exists a normal operator $N$ acting on a Hilbert space $\mathscr{Y}$, which contains $\mathscr{H}$, such that $N x=S x$ for all $x$ in $\mathscr{H}$. $N$ is called the minimal normal extension (abbreviated mne.) of $S$ when $\mathscr{\varkappa}$ is the only closed subspace containing $\mathscr{L} C$ that reduces $N$. This is equivalent to saying that the closure of the linear manifold

$$
\left\{\sum_{j=0}^{n} N^{*^{j}} x_{j} \mid x_{j} \in \mathscr{K}, n \text { a nonnegative integer }\right\}
$$

is all of $\mathscr{K}$. (For the elementary properties of subnormal operators consult $[2,5]$.

If $K$ is a compact set in the plane then $C(K)$ denotes the continuous functions on $K$ and $\mathscr{A}(K)$ is the collection of functions $f$ analytic on some open set $G(f) \supset K . \quad P(K)$ and $R(K)$ are the uniform closures of the polynomials and rational functions with poles off $K$, respectively. Further, $\partial K$ and int $K$ denote the boundary and interior of $K$, respectively. $\hat{K}$ designates the polynomial convex hull of $K$. [3, p. 66].

The set of bounded operators on a (complex) Hilbert space $\mathscr{Z}$ is denoted by $\mathscr{B}(\mathscr{H})$ and $\sigma(T)$ represents the spectrum of any operator $T$ belonging to $\mathscr{B}(\mathscr{H})$. Finally, $C$ denotes the complex numbers and $\boldsymbol{N}$ denotes the nonnegative integers.

2. The problem. Throughout the rest of this paper it will be 
assumed that $S$ is a subnormal operator on $\mathscr{H}$ with its mne. $N$ on $\mathscr{K}$. It is well-known that $\sigma(S) \supset \sigma(N)$ and, in fact, $\sigma(S)$ is obtained by "filling in" some of the holes of $\sigma(N)$. (See [2].) Moreover, $\sigma(S)$ and $\sigma(N)$ are spectral sets for $S$ and $N$, respectively. (Consult [9] for the basic properties of spectral sets.)

Consequently, for each $f$ in $R(\sigma(S))$ an operator $f(S)$ belonging to $\mathscr{B}(\mathscr{H})$ may be defined. Furthermore, a normal operator $f(N)$ (in $\mathscr{B}(\mathscr{K})$ ) is defined because $R(\sigma(S)) \subset R(\sigma(N))$. For these functions $f$, it is easy to show that $f(N) \mathscr{H} \subset \mathscr{H}$ and that

$$
\left.f(N)\right|_{\mathscr{C}}=f(S) \text {. }
$$

$\left(\left.f(N)\right|_{\mathscr{L}}\right.$ means the restriction of $f(N)$ to $\left.\mathscr{H}.\right)$ It now follows that $f(S)$ is subnormal.

Moreover, for functions $f$ in $\mathscr{A}(\sigma(S))$ or $P(\sigma(S))$ we can define a normal (subnormal) operator $f(N)(f(S)$ ) such that (2.1) holds. This follows because $P(\sigma(S))$ is a uniformly closed subalgebra of $R(\sigma(S))$ and each function $f$ belonging to $\mathscr{A}(\sigma(S))$ may be approximated uniformly on $\sigma(S)$ by rational functions with poles off $\sigma(S)$. (See Runges Theorem [7, p. 256].)

With this background a natural question arises.

Question 2.2. For which function $f$ belonging to $R(\sigma(S)$ ) (or $\mathscr{A}(\sigma(S))$ or $P(\sigma(S)))$ is $f(N)$ the minimal normal extension of $f(S)$ ?

In the next section we present the approximation theorems that will be the foundation for the answer to (2.2). Before this is done, it is shown that the spectrum of the mne. of $f(S)$ is $\sigma(f(N))$.

THEOREM 2.3. Let $f$ be a bounded Borel function on $\sigma(N)$ such that $f(N) \mathscr{H} \subset \mathscr{H}$ and set $f(S)=f(N)_{\mathscr{P}} \mid$. Then the spectrum of the mne. of $f(S)$ is equal to the spectrum of $f(N)$.

Proof. Let $\mathscr{C}$ denote the closure of the linear manifold

$$
\left\{\sum_{j=0}^{n} f(N)^{* j} x_{j} \mid x_{j} \in \mathscr{H}, n \in N\right\}
$$

and set $T=\left.f(N)\right|_{\mathscr{A}}$. Its clear that $T$ (on $\mathscr{M}$ ) is the mne. of $f(S)$ and $N \mathscr{C} \subset \mathscr{C l}$ because $N f(N)^{*}=f(N)^{*} N$. Set $S_{1}=\left.N\right|_{\mathscr{M}}$ and observe that $N$ is the mne. of $S_{1}$ because $\mathscr{H} \subset \mathscr{M} \subset \mathscr{K}$.

Since $f(N)=\left.T \oplus f(N)\right|_{\mathscr{A} \perp}$ we have the obvious inclusion $\sigma(T) \subset$ $\sigma(f(N))$. Suppose $\sigma(f(N)) \backslash \sigma(T)$ is nonempty and let $\lambda$ be an element of this set. Choose a relatively open set $G$ in $\sigma(f(N))$ such that $\lambda \in G$ and $\bar{G}$ (the closure of $G$ ) has an empty intersection with $\sigma(T)$. Let $E$ be the spectral measure for $f(N)$. In other words $f(N)=$ 
$\int z d E(z)$. Set $P$ equal to the projection from $\mathscr{K}$ onto $\mathscr{C}$ and observe $P E(\Delta)=E(\Delta) P$ for all Borel sets $\Delta$. By the uniqueness of spectral measures it is easy to verify that the spectral measure, $\widetilde{E}$, of $T$ is given by $\widetilde{E}(\Delta)=P E(\Delta) P=P E(\Delta)$.

Because $\bar{G} \cap \sigma(T)=\square$ we have $\widetilde{E}(\bar{G})=0$. Therefore $0=P E(\bar{G})=$ $E(\bar{G}) P$ so that the range of $E(\bar{G})$ is orthogonal to $\mathscr{C l}$. Now $G$ a nonempty relatively open set in $\sigma(f(N))$ implies $E(\bar{G}) \neq 0$ which is a contradiction of the fact that $N$ is the mne. of $S_{1}$. Therefore $\sigma(f(N)) \backslash \sigma(T)=\square$. The proof is now complete.

\section{Approximation theorems.}

THEOREM 3.1. Let $K$ be a compact set in the plane and $f$ an analytic function on an open set $G \supset K$. If $f$ is not constant on any component of $G$ then the linear span of the set

$$
\mathscr{F}=\left\{\bar{f}^{n} f^{m} \boldsymbol{z}^{p} \mid n, m, p \in N\right\}
$$

is uniformly dense in $C(K)$.

The proof of this theorem as well as the proofs of Theorem 3.2 and Theorem 3.4 rely on Bishop's Generalized Stone-Weierstrass Theorem. (See $[1,3,4]$.) Since their proofs are very similar, only the proof of Theorem 3.4 will be presented here. (Consult the remarks at the end of the proof of Theorem 3.4.)

By the Maximum-Modulus Principle every function $f$ belonging to $P(K)$ may be extended to a function in $P(\hat{K})$. This identification is made in the following theorem.

\section{THEOREM 3.2. Let}

1. $K$ be a compact set in the plane and $\left\{G_{\imath}\right\}$ be the sequence of components of int $\hat{K}$,

2. $f$ belong to $P(K)$ and $\mathscr{U}$ denote the uniformly closed algebra generated in $C(K)$ by $\bar{f}$ and the polynomials.

If $\left.f\right|_{\partial G_{i}} \neq$ constant for all $i$ then $\mathscr{U}=C(K)$.

DeFinition 3.3. Let $K$ be a compact set in the plane. We say that $f$ is a minimal function on $K$ if $f \in R(K)$ and for every complex number $c$

$$
R\left(L_{c}\right)=C\left(L_{c}\right)
$$

where $L_{c}=\{z \in K \mid f(z)=c\}$.

Before we present some examples of minimal functions, we show 
why they are interesting.

Theorem 3.4. Let $K_{0}$ be a compact subset in the plane, $\left\{G_{2}\right\}$ denote the sequence of bounded components of $C \backslash K_{0}$, and let

$$
K_{1}=K_{0} \cup\left(\bigcup_{i \in I} G_{i}\right)
$$

where $I$ is an arbitrary subset of the positive integers.

If $f$ is a minimal function on $K_{1}$ and $\mathscr{Q}$ is the uniformly closed algebra generated in $C\left(K_{0}\right)$ by $\bar{f}$ and $R\left(K_{1}\right)$ then $\mathscr{U}=C\left(K_{0}\right)$.

Proof. Let $F$ be a maximal set of antisymmetry of $\mathscr{U}$. Now $f$ and $\bar{f}$ belong to $\mathscr{U}$ so there exists a constant $c$ such that

$$
F \subset\left\{z \in K_{0} \mid f(z)=c\right\} \subset\left\{z \in K_{1} \mid f(z)=c\right\} .
$$

By Bishop's Theorem it suffices to show $C(F)=\left.\mathscr{U}\right|_{F}$.

Since $f$ is minimal on $K_{1}$ we clearly have $R(F)=C(F)$. Therefore it suffices to show $\left.\mathscr{U}\right|_{F} \supset R(F)$. Since $\left.\mathscr{\mathscr { C }}\right|_{F}$ is closed in $C(F)$ the last statement will follow if it can be demonstrated that $\left.r \in \mathscr{K}\right|_{F}$ for all rational functions $r$ with poles off $F$. Before we prove the latter the following observation is made.

Claim one. $f$ minimal on $K_{1}$ implies $f$ is not constant on any component of int $K_{1}$.

Suppose the contrary, say there exists a component $G$ of int $K_{1}$ such that $f(G)=c_{0}$. If $z \in G$ then for every neighborhood $U$ of $z$ it follows that $L_{c_{0}} \cap \bar{U}$ has nonempty interior. Hence $R\left(L_{c_{0}} \cap \bar{U}\right) \neq$ $C\left(L_{c_{0}} \cap \bar{U}\right)$ because every function belonging to the former set is analytic on $\operatorname{int}\left(L_{c_{0}} \cap \bar{U}\right)$. This contradicts the fact that $f$ is minimal on $K_{1}$.

Now fix a rational function $r$ with poles off $F$. Let the sequence of components of $C \backslash F$ be denoted by $\left\{U_{\imath}\right\}$ where $U_{0}$ denotes the unbounded component and let the sequence of $\boldsymbol{C} \backslash K_{1}$ be denoted by $\left\{\mathscr{O}_{j}\right\}$ where $\mathscr{O}_{0}$ denotes the unbounded component. Because $C \backslash K_{1} \subset$ $C \backslash F$, for each $j$ there exists a unique $i_{j}$ such that $O_{j} \subset U_{i_{j}}$. We wish to show that the map $\mathbb{Q}_{j} \rightarrow U_{i_{j}}$ is an onto map from $\left\{\mathscr{O}_{j}\right\}$ to $\left\{U_{i}\right\}$. Therefore we want to prove.

Claim two. For each $i$ there exists a $j$ such that $\mathcal{O}_{j} \subset U_{i}$.

Suppose that some $U_{i}$ does not contain any $\mathcal{O}_{j}$. Then because $C \backslash K_{1}=\cup_{j} \mathcal{O}_{j}$ and $\mathscr{O}_{0} \cup U_{0}$ it follows that $U_{i} \subset K_{1}$. But $\partial U_{i} \subset F$ and so $f$ is constant on some component of int $K_{1}$ which contradicts claim one.

Hence, each $U_{i}$ contains a point of the complement of $K_{1}$. Since 
$r$ is analytic on some open set $F_{1}$ and since $\mathscr{C}$ contains the rational functions with poles of $K_{1}$, Runge's Theorem gives $\left.r \in \mathscr{C}\right|_{F}$. This completes the proof.

Remarks. (1) In the last section of this paper an example is given to show that (notation as above) if $\mathscr{U}^{\prime}$ is the uniformly closed algebra generated in $C\left(K_{0}\right)$ by $\bar{f}$ and $P\left(K_{1}\right)$ then $\mathscr{U}^{\prime}$ may fail to be $C\left(K_{0}\right)$. (2) To prove Theorem 3.1 observe that any maximal set of antisymmetry of the uniformly closed algebra generated by $\mathscr{F}$ must consist of only finite number of points by the conditions on $f$. Therefore, since $\mathscr{F}$ contains the polynomials, each maximal set of antisymmetry must consist of only one point. (3) To prove Theorem 3.2 observe that each maximal set of antisymmetry $F$ must be nowhere dense and $C \backslash F$ must be connected. Therefore $C(F)=P(F)$ by Lavrentiev's Theorem. (Consult [3, p. 48].)

This section of the paper is concluded by giving some examples of minimal functions. Let $f$ be an entire function which is not constant. Then $f \in R(K)$ for any compact set $K$ and for any constant $c$ the level set $L_{c}=\{z \in K \mid f(z)=c\}$ consists of a finite number of points and therefore $f$ is minimal on $K$.

If $f \in P(K)$ and $f$ is not constant on any components of int $\hat{K}$ it is easy to show $f$ is minimal on $K$. (In fact, one gets $P\left(L_{c}\right)=$ $C\left(L_{c}\right)$ for any level set $L_{c}$.) Consequently, (by the first claim in the proof of Theorem 3.4) if $f \in P(K)$ then a necessary and sufficient condition that $f$ be minimal on $\hat{K}$ is that $f$ is not constant on any component of int $\hat{K}$.

LEMMA 3.5. Let $K$ be a compact subset in the plane satisfying either of the following conditions:

(a) The planar measure of $\partial K$ is equal to zero,

(b) $R(K)$ is a Dirichlet algebra. (See [3, p. 34].)

If $f \in R(K)$ and $f$ is not constant on any component of int $K$ then $f$ is minimal on $K$.

Proof. First case. $f \in R(K)$ and $\partial K$ has planar measure zero. Fix a constant $c$ and the level set $L_{c}=\{z \in K \mid f(z)=c\}$. Then

$$
L_{c}=\left\{L_{c} \cap \operatorname{int} K\right\} \cup\left\{L_{c} \cap \partial K\right\} \text {. }
$$

$\left\{L_{c} \cap\right.$ int $\left.K\right\}$ is countable by the hypothesis on $f$ and $\left\{L_{c} \cap \partial K\right\}$ has planar measure zero by the hypothesis on $\partial K$. Therefore, the planar measure of $L_{c}$ is zero and by the Hartog-Rosenthal Theorem [3] we have $R\left(L_{c}\right)=C\left(L_{c}\right)$.

Second case. Again fix a constant $c$ and write $L_{c}$ as in (3.6). Since every point of $\left\{L_{c} \cap\right.$ int $\left.K\right\}$ is an isolated point (because $f$ is 
not constant on any component of int $K$ ) each such point is clearly a peak point for $R\left(L_{c}\right)$. Since $R(K)$ is a Dirichlet algebra every point in $\left\{L_{c} \cap \partial K\right\}$ is a peak point of $R(K)$ and hence a peak point of $R\left(L_{c}\right)$. Thus every point of $L_{c}$ is a peak point for $R\left(L_{c}\right)$ so by $[3$, p. 54]

$$
R\left(L_{c}\right)=C\left(L_{c}\right)
$$

\section{Answers to question 2.2.}

TheOREM 4.1. Let $S$ be a subnormal operator on $\mathscr{H}$ and $N$ be its mne. on $\mathscr{K}$. If $f$ is analytic on an open set $G \supset \sigma(S)$ such that $f$ is not constant on any component of $G$ then $f(N)$ is the mne. of $f(S)$.

Proof. Let $\mathscr{C}$ denote the closure of the linear manifold

$$
\left\{\sum_{j=0}^{n} f(N)^{*^{j}} x_{j} \mid x_{j} \in \mathscr{H}, n \in N\right\} .
$$

It suffices to show $\mathscr{C}=\mathscr{K}$.

Observe that $N, f(N)$ and $f(N)^{*}$ all leave $\mathscr{M}$ invariant. Therefore any operator $T$ in the norm closed algebra $\mathscr{A}$ generated by these operators and the identity leaves $\mathscr{C}$ invariant. By the Spectral Theorem the algebra $\mathscr{A}$ is $*$ isometrically isomorphic to the uniformly closed algebra $\mathscr{C}$ in $C(\sigma(N))$ generated by $\bar{f}, f, z$ and 1. By Theorem 3.1, $\bar{z} \in \mathscr{U}$, hence $N^{*} \in \mathscr{A}$. Therefore $N^{*} \mathscr{C l} \subset \mathscr{L}$. Since $\mathscr{H} \subset \mathscr{C} \subset \mathscr{K}$, by minimality $\mathscr{C}=\mathscr{K}$. This finishes the proof.

As an immediate consequence of the last proof we have

Corollary 4.2. If $N$ is a normal operator, $f \in \mathscr{A}(\sigma(N))$ and $f$ is not constant on any component of its domain then the commutative $C^{*}$ algebra generated by $N$ and the identity is equal to the commutative algebra generated by $f(N), f(N)^{*}, N$ and the identity.

Just as Theorem 4.1 follows directly from Theorem 3.1 the following two theorems follow directly from the other two approximation theorems in section three.

THEOREM 4.3. Let $S$ be a subnormal operator on $\mathscr{H}$ and $\mathscr{N}$ be its mne. on $\mathscr{K}$. Let $\left\{G_{i}\right\}$ denote the sequence of components of int $\widehat{\sigma(S)}$. If $f \in P(\sigma(S))$ and $f$ is not constant on any $G_{i}$ then $f(N)$ is the mne. of $f(S)$.

THeorem 4.4. Let $S$ be a subnormal operator on $\mathscr{H}$ with mne. 
$N$ on $\mathscr{K}$. If $f$ is a minimal function on $\sigma(S)$ then $f(N)$ is the mne. of $f(S)$.

REMARK. The proof of Theorem 4.4 follows exactly like the proof of Theorem 4.1 except one replaces Theorem 3.1 with Theorem 3.4 using $K_{0}=\sigma(N)$ and $K_{1}=\sigma(S)$.

Using Theorem 4.3 it is possible to describe the mne. of $f(S)$ for an arbitrary function $f$ belonging to $P(\sigma(S))$. More precisely; let the sequence of components of int $\widehat{\sigma(S)}$ be denoted by $\left\{\mathcal{O}_{i}\right\}_{i=0}^{\infty}$ (if the sequence is finite the procedure is the same) and let $I_{f}$ denote the set of positive integers such that if $i \in I_{f}$ then $\left.f\right|_{o_{i}}=$ constant $=$ $\alpha_{i}$, and if $i \notin I_{f}$ then $\left.f\right|_{o_{i}} \neq$ constant.

Using $\left[8\right.$, p. 296] we can find closed subspaces $\mathscr{H}_{i} i=0,1,2, \cdots$ such that $\mathscr{H}_{i} \perp \mathscr{H}_{j}$ for $i \neq j, \sum_{0}^{\infty} \oplus \mathscr{H}_{\imath}=\mathscr{H}$, jeach $\mathscr{H}_{i}$ reduces $S$ and if $S_{i}=\left.S\right|_{\mathscr{C}_{i}}$ then $S=S_{0} \oplus\left(\sum_{1}^{\infty} \oplus S_{i}\right)$ with respect to $\mathscr{H}=\sum_{0}^{\infty} \oplus \mathscr{H}_{i}$. Moreover we can choose the $\mathscr{H}_{i}$ 's such that (a) $S_{0}$ is normal and $\sigma\left(S_{0}\right) \subset \partial \widehat{\sigma(S)}$ and $(b) \bar{O}_{i}$ is a spectral set for $S_{i}$.

Without loss of generality we can assume that the mne. of $S$ is $N=S_{0} \oplus\left(\sum_{1}^{\infty} \oplus N_{i}\right)$ with respect to $\mathscr{K}=\mathscr{H}_{0} \oplus\left(\sum_{1}^{\infty} \oplus \mathscr{K}_{i}\right)$ where $N_{i}$ (on $\mathscr{K}_{i}$ ) is the mne. of $S_{i}$ (on $\mathscr{K}_{i}$ ) for $i=1,2 \cdots$.

Claim. $f\left(N_{2}\right)$ is the mne. of $f\left(S_{i}\right)$ for all $i \notin I_{f}$. First observe that $f\left(N_{i}\right)$ and $f\left(S_{i}\right)$ are defined because $f \in P(\sigma(S)) \subset P\left(\sigma\left(S_{i}\right)\right)$.

Now fix a complex number $c$ and its associated level set $L_{c}=$ $\left\{z \in \sigma\left(S_{i}\right) \mid f(z)=c\right\} \subset\left\{z \in \bar{O}_{i} \mid f(z)=c\right\} \equiv B$.

Since $f$ is not constant on $O_{i}$ and $R(\widehat{\sigma(S)})=P(\sigma(S))$ is a Dirichlet algebra, by the same methods used in the proof of Lemma 3.5, it follows that $R(B)=C(B)$ which implies $R\left(L_{c}\right)=C\left(L_{c}\right)$. This in turn implies that $f$ is minimal on $\sigma\left(S_{i}\right)$. This establishes the claim.

It is now clear that the mne. of $f(S)$ is $f\left(S_{0}\right) \oplus\left(\sum_{i \notin I_{f}} \oplus f\left(N_{i}\right)\right) \oplus$ $\left(\sum_{i \in I_{f}} \oplus \alpha_{i}\right)$ with respect $\mathscr{H}_{0} \oplus\left(\sum_{i \notin I_{f}} \oplus \mathscr{K}_{i}\right) \oplus\left(\sum_{i \in I_{f}} \oplus \mathscr{H}_{i}\right)$.

What is the mne. of $f(S)$ for an arbitrary function $f$ belonging to $R(\sigma(S))$ ? If $R(\sigma(S))$ is a Dirichlet algebra the question has a natural solution. Proceed exactly as above except let $\left\{\mathcal{O}_{i}\right\}_{1}^{\infty}$ be the sequence of components of int $\sigma(S)$. Since $R(\sigma(S))$ is a Dirichlet algebra the nontrivial Gleason parts of $R(\sigma(S))$ are precisely the $O_{i}$ 's.

Now we can use Mlak's results [6] to find subspaces $\mathscr{C}_{i}$ whose relationships to the $\mathcal{O}_{i}^{\prime}$ 's are precisely like the case above except that $\sigma\left(S_{0}\right) \subset \partial \sigma(S)$. The calculation above now carries over.

The general case (no assumption on $R(\sigma(S))$ ) still remains open.

5. Some consequences. Recall that an operator $T \in \mathscr{B}(\mathscr{K})$ is reductive if and only if every invariant subspace of $T$ reduces $T$. 
CoRollary 5.1. Let $N$ be a normal operator and $f \in P(\sigma(N))$

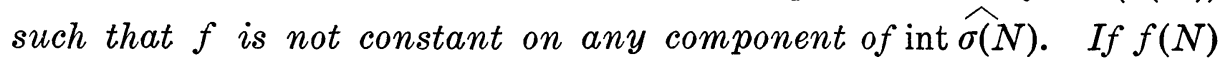
is a reductive operator then $N$ is a reductive operator.

Proof. If $N$ (on $\mathscr{K}$ ) is not a reductive operator then $N$ must be the mne. of some (nonnormal) subnormal $S$ (on $\mathscr{H}$ ). By Theorem $4.3 f(N)$ is the mne. of $f(S)$, so $\mathscr{H}$ is a nonreducing invariant subspace for $f(N)$.

CoROLlary 5.2. If $f$ is a minimal function on $\sigma(S)$ and $f(S)$ is normal then $S$ is normal.

A subnormal operator $S$ is called completely subnormal if $S$ has no nonzero reducing subspace on which it is normal.

Lemma 5.3. Let $N$ (on $\mathscr{K}$ ) be the mne. of $S$ (on $\mathscr{H}$ ). $S$ is completely subnormal if and only if $N^{*}$ is the mne. of $\left.N^{*}\right|_{\mathscr{*}}$.

Proof. Suppose $N^{*}$ is not the mne. of $\left.N^{*}\right|_{\mathscr{H} \perp} . \quad\left(N^{*} \mathscr{H}^{\perp} \subset \mathscr{H}^{\perp}\right.$ because $N \mathscr{H} \subset \mathscr{H}$.) Then there exists a closed subspace $\mathscr{C}$ such that $\mathscr{H}^{\perp} \subset \mathscr{M} \subsetneq \mathscr{K}, N^{*} \mathscr{M} \subset \mathscr{M}$ and $N \mathscr{M} \subset \mathscr{M}$. Therefore $0 \neq$ $\mathscr{M}^{\perp} \subset \mathscr{H}$ and $\left.N\right|_{\mathscr{L}^{\perp}}=\left.S\right|_{\mathscr{N} \perp}$ is normal which implies $S$ is not completely subnormal. By reversing the above argument the proof is completed.

CoROLlaRY 5.4. If $S$ is completely subnormal and $f$ is a minimal function on $\sigma(S)$ then $f(S)$ is completely subnormal.

Proof. By Lemma 5.3 it suffices to show $f(N)^{*}$ is the mne. of $\left.f(N)^{*}\right|_{\mathscr{L} \perp}$. It is easy to show that $\overline{\sigma(S)}=\sigma\left(\left.N^{*}\right|_{\mathscr{H} \perp}\right)$.

Now define a function $g$ on $\sigma\left(\left.N^{*}\right|_{\mathscr{C}}\right)$ by $g(z)=\overline{f(\bar{z})}$ for $z \in \overline{\sigma(S)}$. Because $f$ is minimal on $\sigma(S), g$ is minimal on $\overline{\sigma(S)}$. Therefore $g\left(N^{*}\right)=f(N)^{*}$ is the mne. of $\left.f(N)^{*}\right|_{\mathscr{O}}$.

6. An example. The purpose of this section is to give an example of a compact set $K$ and a minimal function $\varphi$ on $K$ such that the uniformly closed algebra $\mathscr{U}$ generated by $\bar{\varphi}$ and $P(K)$ is not $C(K)$. (See Theorem 3.4.)

Let $F$ be the cornucupia (that is, the closed unit disk, $\bar{D}$, with a ribbon which winds around from the outside and clusters on the boundary of the disk, $\partial D$ ). (For a picture, see [3, p. 152].) Let $K=F$ with the interior of the disk deleted. That is, $K=\{$ ribbon $U$ $\partial D$. Since the interior of $K$ is simply connected we may choose a conformal map $\varphi$ from int $K$ onto the open unit disk $D$. 
By [7, p. 280] we extend $\varphi$ to be a one-to-one and continuous function on $A \cup$ int $K$, where $A$ equals the boundary of the ribbon with $\partial D$ delated. Since $\Lambda$ is connected, $\varphi(\Lambda)$ is an arc on the unit circle.

Let $\partial D \backslash \varphi(\Lambda)=[\alpha, \beta]$. (It is clear that $\partial D \backslash \varphi(\Lambda)$ is a closed arc.) Since $\varphi^{-1}$ has radial limits almost everywhere, because $\varphi^{-1}$ is a bounded analytic function in the open disk, it can be shown that $[\alpha, \beta]$ consists of exactly one point, say $[\alpha, \beta]=\{\lambda\}$. We now exend the definition of $\varphi$ to all of $F$ by defining $\varphi$ on $\bar{D}$ to be equal to $\lambda$ where $\{\lambda\}=\partial D \backslash \varphi(\Lambda)$. A straightforward sequence argument shows $\phi$ is continuous on $F$, and by construction $\varphi$ is analytic on int $F$. Therefore by Mergelyan's Theorem [3, p. 48] $\varphi \in P(K)$. It now follows that $\phi$ is minimal on $K$.

What is the uniform algebra $\mathscr{C}$ generated in $C(K)$ by $\bar{\varphi}$ and the polynomials? It is easy to show that the maximal sets on antisymmetry for $\mathscr{U}$ are precisely the singleton sets $\{w\}$ for all $w$ in $K \backslash \partial D$ together with the set $\partial D$. It then follows that a function $f$ is an element of $\mathscr{Q}$ if and only if $f \in C(K)$ and $f$ can be extended to a function $\hat{f} \in C(\hat{K})(=C(F))$ such that $\left.\hat{f}\right|_{\bar{D}} \in P(\bar{D})$.

\section{REFERENCES}

1. E. Bishop, A generalization of the Stone-Weierstrass theorem, Pacific J. Math., 11 (1961), 777-783.

2. J. Bram, Subnormal Operators, Duke Math. J., 22 (1955), 75-94.

3. T. Gamelin, Uniform Algebras, Prentice-Hall, Englewood Cliffs, 1969.

4. I. Glicksberg, Measures orthogonal to algebras and sets of antisymmetry, Trans. Amer. Math. Soc., 105 (1962), 415-435.

5. P. Halmos, A Hilbert Space Probelm Book, Van Nostrand, Princeton, 1967.

6. W. Mlak, Partitions of spectral sets, Ann. Pol. Math., 25 (1972), 273-280.

7. W. Rudin, Real and Complex Analysis, McGraw-Hill, New York, 1966.

8. D. Sarason, On spectral sets having connected complement, Acta Sci. Math. (Szeged), 26 (1965), 289-299.

9. J. von Neumann, Eine spectral theorie für allgemeine operatoren eines unitären raumes, Math. Nachr., 4 (1951), 258-281.

Received July 28, 1975. This paper is part of the author's Ph. D. thesis written under the supervision of John B. Conway, at Indiana University.

INDIANA UNIVERSITY

AND

Virginia Polytechnic Institute and State University 



\section{PACIFIC JOURNAL OF MATHEMATICS}

EDITORS

RICHARD ARENS (Managing Editor)

University of California

Los Angeles, California 90024

R. A. BEAUMONT

University of Washington

Seattle, Washington 98105
J. DugundJI

Department of Mathematics

University of Southern California

Los Angeles, California 90007

D. Gilbarg and J. Milgram

Stanford University

Stanford, California 94305

\section{ASSOCIATE EDITORS}

E. F. BECKENBACH

B. H. NeumanN

F. WOLF

K. YosHIDA

\section{SUPPORTING INSTITUTIONS}

UNIVERSITY OF BRITISH COLUMBIA

UNIVERSITY OF SOUTHERN CALIFORNIA

CALIFORNIA INSTITUTE OF TECHNOLOGY

UNIVERSITY OF CALIFORNIA

STANFORD UNIVERSITY

UNIVERSITY OF TOKYO

MONTANA STATE UNIVERSITY

UNIVERSITY OF UTAH

UNIVERSITY OF NEVADA

WASHINGTON STATE UNIVERSITY

NEW MEXICO STATE UNIVERSITY

UNIVERSITY OF WASHINGTON

OREGON STATE UNIVERSITY

UNIVERSITY OF OREGON

OSAKA UNIVERSITY

AMERICAN MATHEMATICAL SOCIETY
NAVAL WEAPONS CENTER

Printed in Japan by International Academic Printing Co., Ltd., Tokyo, Japan 


\section{Pacific Journal of Mathematics}

\section{Vol. 63, No. $1 \quad$ March, 1976}

Ralph Artino, Gevrey classes and hypoelliptic boundary value problems ....... 1

B. Aupetit, Caractérisation spectrale des algèbres de Banach commutatives .... 23

Leon Bernstein, Fundamental units and cycles in the period of real quadratic

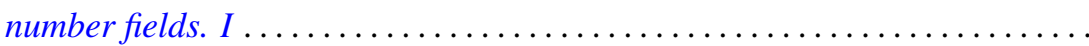

Leon Bernstein, Fundamental units and cycles in the period of real quadratic number fields. II.................................... 63

Robert F. Brown, Fixed points of automorphisms of compact Lie groups ........

Thomas Ashland Chapman, Concordances of noncompact Hilbert cube

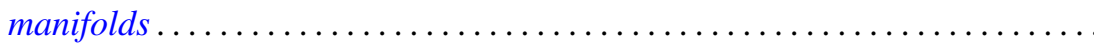

William C. Connett, V and Alan Schwartz, Weak type multipliers for Hankel

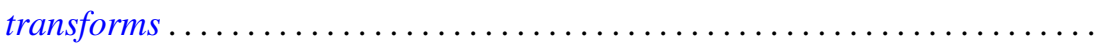

John Wayne Davenport, Multipliers on a Banach algebra with a bounded approximate identity .....................................

Gustave Adam Efroymson, Substitution in Nash functions ................ 137

John Sollion Hsia, Representations by spinor genera ..................

William George Kitto and Daniel Eliot Wulbert, Korovkin approximations in

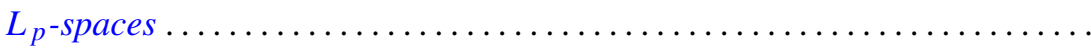

Eric P. Kronstadt, Interpolating sequences for functions satisfying a Lipschitz. condition ...........................................

Gary Douglas Jones and Samuel Murray Rankin, III, Oscillation properties of certain self-adjoint differential equations of the fourth order...

Takaŝi Kusano and Hiroshi Onose, Nonoscillation theorems for differential

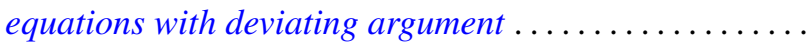

David C. Lantz, Preservation of local properties and chain conditions in commutative group rings. ...

Charles W. Neville, Banach spaces with a restricted Hahn-Banach extension property....

Norman Oler, Spaces of discrete subsets of a locally compact group ...

Robert Olin, Functional relationships between a subnormal operator and its minimal normal extension.

Thomas Thornton Read, Bounds and quantitative comparison theorems for nonoscillatory second order differential equations ...... .

Robert Horace Redfield, Archimedean and basic elements in completely distributive lattice-ordered groups...

Jeffery William Sanders, Weighted Sidon sets

Aaron R. Todd, Continuous linear images of pseudo-complete linear topological spaces.

J. Jerry Uhl, Jr., Norm attaining operators on $L^{1}[0,1]$ and the Radon-Nikodým property.

William Jennings Wickless, Abelian groups in which every endomorphism is a left multiplication. 